\title{
La evolución de la producción y de la inversión en el sector petrolero y gasífero en Argentina
}

\section{The evolution of the production and the investment in the oil and gas sector in Argentina}

\author{
Matías Nicolás VILLARROEL, nicolasvillarroel5 @gmail.com \\ Daniel SCHINELLI, daniel.schinelli@ speedy.com.ar \\ Carlos VACCA, carlos.vacca@speedy.com.ar
}

\begin{abstract}
Universidad Nacional de la Patagonia Austral, Unidad Académica Río Gallegos, Instituto de Trabajo, Economía y Territorio (I.T.E.T)

Av. Gobernador Gregores y Piloto "Lero" Rivera. Río Gallegos, Santa Cruz, Argentina
\end{abstract}

Recibido: 13/03/2018. Aceptado: 29/10/2018

\section{RESUMEN}

Este estudio plantea como ha evolucionado la producción e inversión del Sector Petrolero y Gasífero en Argentina. Dichos sectores que forman parte de la matriz productiva de la Provincia de Santa Cruz son considerados una importante fuente de recursos para la región. El mismo es analizado a través de los niveles de producción de ambos sectores en los últimos 17 años y la inversión realizada en los mismos en el período 2006-2017. La metodología utilizada en este estudio se basa en recolección de datos, elaboración de gráficos, relacionando los datos relevantes con hechos económicos mundiales y nacionales que permitan una mejor interpretación de la información obtenida y finalmente la elaboración de conclusiones en donde podremos observar el escenario en el cual nos encontramos y cuáles son los interrogantes que nos plantearemos.

Palabras clave: Petróleo; Gas; Inversión; Producción; Evolución.

\begin{abstract}
This study raises has evolved as production and investment of oil and gas sector in Argentina. These sectors are part of the productive matrix of the Province of Santa Cruz are considered an important resource for the region. The same is analyzed through production levels of both sectors in the past 17 years and the investment in them in the period 2006-2017. The methodology used in this study is based on data collection, charting the relevant data relating to global and national economic events that allow better interpretation of the information obtained and finally drawing conclusions on where we can see the scene in which we stand and what are the questions we ask.
\end{abstract}

Key words: Oil; Gas; Investment; Production; Evolution. 


\section{INTRODUCCIÓN}

El presente informe fue realizado en el marco del Proyecto de Investigación: "Dinámica Productiva en Santa Cruz: Su transformación e incidencia en el empleo", el cual continúa la línea de investigación del proyecto: "Comportamiento de los Sectores Económicos Dinámicos de la Provincia de Santa Cruz en la reconfiguración de la matriz productiva y su impacto en el empleo" finalizado en el año 2016, ambos de la Universidad Nacional de la Patagonia Austral, Unidad Académica Río Gallegos, cuyo director responsable de proyecto es el Mg. Daniel Schinelli.

El mismo tiene como objetivo presentar la evolución de la producción de Petróleo y Gas de la República Argentina en los últimos 17 años (2000-2017).

Asimismo, se agrega un pequeño apartado correspondiente a la evolución de la inversión realizada en dichos sectores. El mismo comprende el período 2006-2017.

Los datos utilizados en la elaboración del informe fueron obtenidos del sitio web oficial de la Secretaría de Energía de la Nación (sistema SESCO) y está actualizado en base al informe presentado el 27 de Febrero del 2013 bajo la denominación: La actividad hidrocarburífera en la Cuenca del Golfo San Jorge: el papel del empresariado regional, realizado por los Licenciados Mariano Prado y Marcelo Robledo, miembros del Proyecto de Investigación "Sectores productivos tradicionales y emergentes en Santa Cruz. Alternativas y continuidades de la dinámica socioeconómica y laboral", dirigido por el Mg. Carlos Vacca.

\section{MARCOS DE REFERENCIA - MARCO HISTÓRICO}

Las investigaciones sobre mercados de trabajo, sectores económicos y alternativas de desarrollo regional en Patagonia Austral constituyen una temática que viene desarrollándose como Línea de Investigación por un equipo consolidado desde el año 1995, con la presentación de importantes resultados científicos y de transferencia que se detallan en otros ítems. En su momento se abordó el estudio del nuevo ordenamiento económico surgido en los años '90 y que generó una significativa modificación de los actores económicos e institucionales, con un importante protagonismo de grupos oligopólicos multinacionales y un reacomodamiento de los actores locales en la gestión de relaciones laborales y de mercado (Mendez Gutiérrez, 1995; Genko y Lipietz, 1997 y 1994, Laurelli y Rofman, 1989, Iñigo Carrera et al., 1994). La configuración clásica de la economía patagónica -ya descripta en anteriores proyectos- se vio afectada por el modelo aperturista mencionado llevando a nuevas injerencias de las Instituciones participantes en la estructura económica regional (Salvia 1999; Rofman 1998; Salvia y Panaia ,1197; Cicciari, 1997). Es preciso señalar que la complejidad de la gestión territorial demanda una sinapsis y sinergia entre sus instituciones que parecen constituir parte de la clave de un desarrollo bien entendido en el territorio dentro de un sistema económico social y tecnológico territorial (Zárate y Artesi, 2007; Boisier, 2001). Las condiciones imperantes a partir de la crisis económica del 2001 generaron una nueva configuración de la economía nacional que impactó significativamente en el funcionamiento de la región. La mayor participación del Estado Nacional y esquemas económicos de corte neokeynesiano generaron una corriente de inversiones que motivaron nuevos comportamientos de los principales sectores económicos. En el caso de Santa Cruz, la explotación de sus recursos hidrocarburíferos permitió mantener un rol de fuerte presencia del Estado Provincial a través de los ingresos marginales percibidos en concepto de regalías, con una fuerte presencia en el mercado laboral. A su vez, los sectores productivos tradicionales y 
emergentes provinciales mostraron una dinámica diferencial permitiendo su reposicionamiento y el desarrollo de nuevos sectores de actividad. La compleja crisis financiera internacional iniciada en el año 2008 generó una reconfiguración de la economía y motivó un papel distinto de las Instituciones. En efecto, desde mediados del 2008, las políticas económicas del gobierno nacional se inscribieron en dicho cuadro de situación, motivando una profundización de los procesos que se habían iniciado posteriormente al año 2002 e innovando con medidas no contempladas hasta ese momento (Basualdo, 2011). La situación de la economía provincial también se vio alterada, afectando a los principales sectores de actividad y generando conflictividad en el funcionamiento del Estado Provincial que debió afrontar distintas crisis sociales y económicas. Como resultado de lo descripto, surge la complejidad de los procesos como materia de análisis vinculada al estudio de la región (García, 1991). Los sistemas están determinados por el tipo de relaciones que se establecen entre los elementos que las componen. Abordar el estudio de los sistemas complejos requiere visiones centradas en las relaciones cualitativas que se dan entre sus elementos (Johnson, 2003). El tránsito de una economía rentista subordinada a la extracción de recursos primarios exportables a otra vinculada con la generación de valor agregado y con la consolidación de encadenamientos productivos sostenidos desde la propia región requiere de tiempo y de importantes esfuerzos institucionales y sociales para su logro (Vacca y Schinelli 2015). El estudio del comportamiento de este sector permite avizorar la evolución del desarrollo económico regional, entendido como proceso reactivador de la economía y dinamizador de la sociedad que mediante el aprovechamiento de los recursos existentes sea capaz de estimular el crecimiento económico, la creación de empleo y la mejora de la calidad de vida de la comunidad. (Alburquerque, 1996).

\section{MATERIALES Y MÉTODOS}

La metodología utilizada en el presente trabajo es la que ofrece la teoría de los "sistemas complejos". El abordaje desde este esquema metodológico implica asumir una perspectiva de estudio interdisciplinaria y multidimensional, tal como aquí se propone desde los campos sociológico, económico y de las ciencias de la administración.

Para el análisis del sector se trabajará con información cualicuantitativa.

En tal sentido se proponen técnicas de recolección de datos y de procesamiento de la información recopilada, de acuerdo a sus particularidades.

\section{RESULTADOS Y DISCUSIÓN}

\section{Producción Nacional de Gas y Petróleo.}




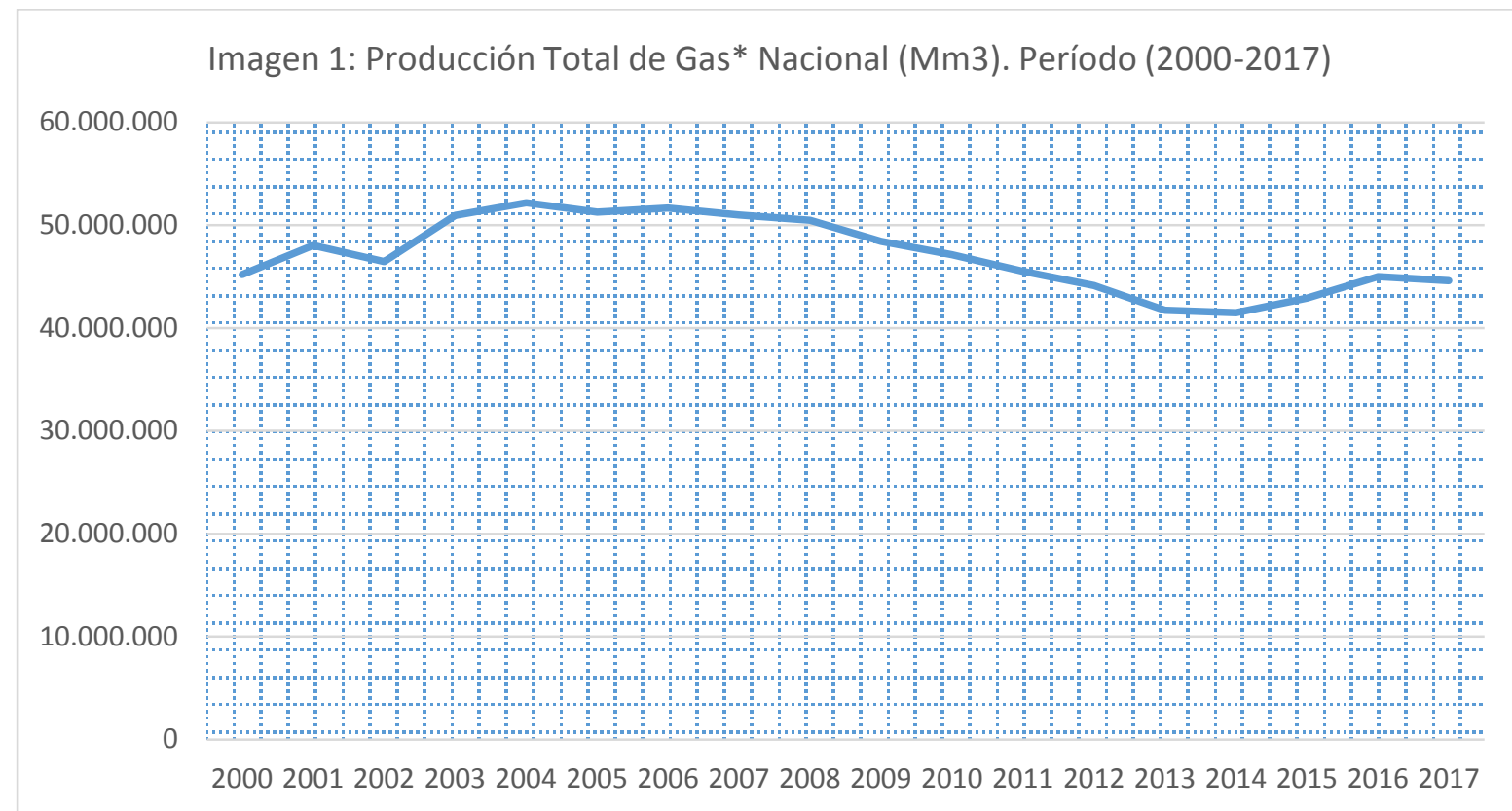

Fuente: Elaboración propia sobre datos de la Secretaría de Energía (Sistema SESCO). Anexo (1).

* El total de gas incluye Gas de Baja, Media y Alta Presión en Miles de metros cúbicos (Mm3).

Si observamos el gráfico anterior se puede deducir que, en los años 2001, 2003 y 2004 se registraron aumentos importantes en la producción de gas. En el caso de la producción de Gas, la tendencia a la baja se produce a partir del año 2004 (52.156.988 Mm3) hasta el 2014 (41.484.024) y si se compara ambos años de referencia la disminución es del $20 \%$.

Un dato a destacar es el siguiente: si bien los niveles de producción han ido disminuyendo con el paso del tiempo, entre el 2013 y el 2014, se registró la disminución más baja de los últimos 15 años. La misma pasó de un nivel de producción de 41.708.289 Mm3 a 41.484.024Mm3, registrándose así una caída del $0,54 \%$.

Por otro lado, se observa un leve aumento de la producción a partir del 2015, registrándose así una suba del 3,42\%, tendencia que se mantiene para el siguiente año (2016) lo cual hace subir la producción en casi 5 puntos $(+4,85 \%)$.

Para el año 2017 y debido al cambio de gobierno reciente en Argentina y en los Estados Unidos, la actividad se mantuvo casi en el mismo nivel, disminuyendo apenas en $392.000 \mathrm{~m} 3$. 


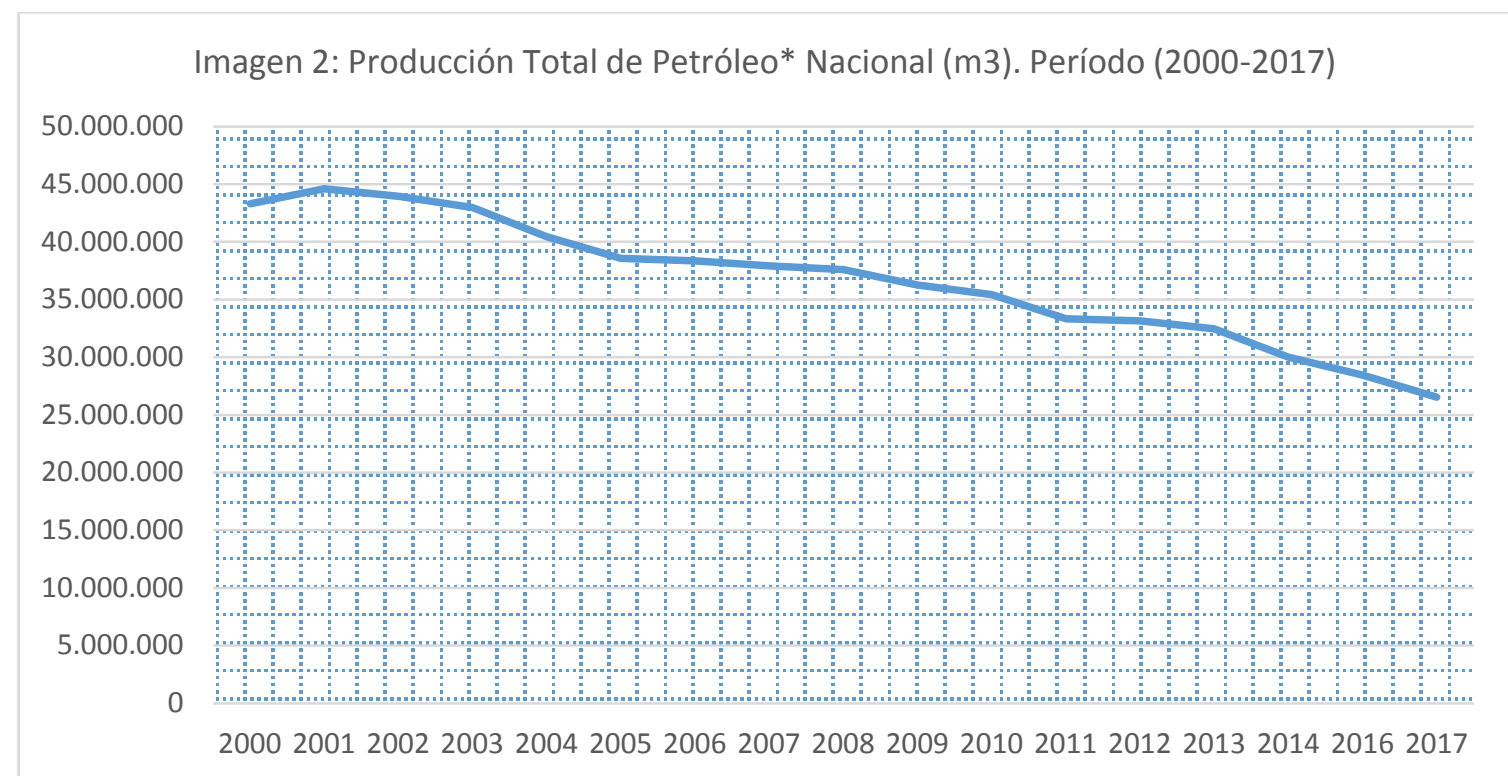

Fuente: Elaboración propia sobre datos de la Secretaría de Energía (Sistema SESCO). Anexo (2). *El total de petróleo incluye Producción Primaria, por Recuperación Asistida y Secundaria en metros cúbicos (m3).

Con respecto a la producción de petróleo en el país se puede observar una disminución de la producción a partir del año 2001, el cual presenta la mayor cantidad en metros cúbicos (44.616.993), hasta el 2014 (30.019.610). Respecto de estos dos años de referencia la disminución es del 32,71\%.

Finalmente se observa que la tendencia a la baja de la producción permanece en los últimos años lo cual es un reflejo del escenario mundial por el que atravesamos.

\section{Inversiones realizadas para la producción de Petróleo y Gas en Argentina.}

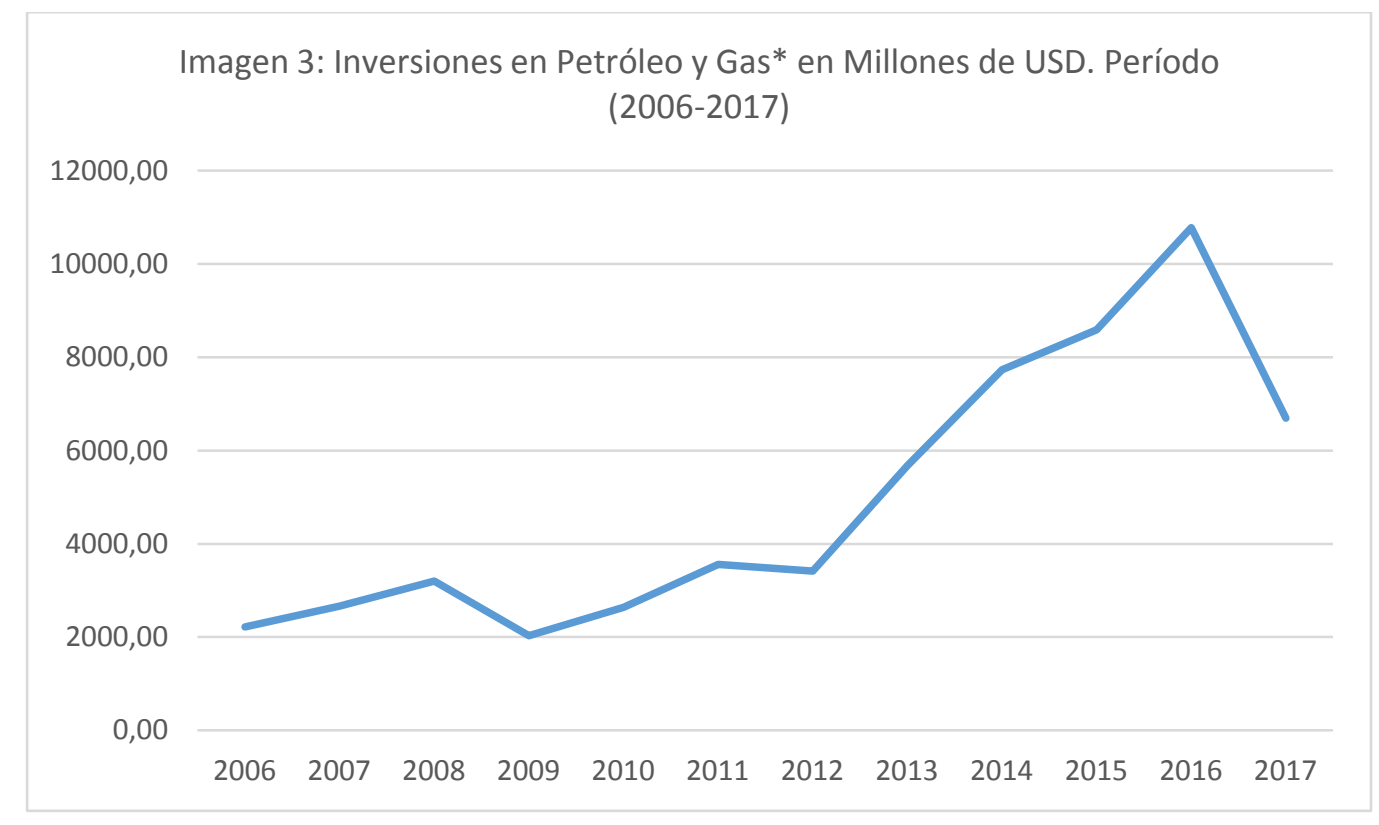

Fuente: Elaboración propia sobre datos de la Secretaría de Energía (Sistema SESCO). Anexo (3) 
*El total de inversiones incluye equipos de bombeo petróleo, instalaciones, laboratorios, equipos de telecomunicaciones, oleoductos, perforación de pozos de gas y petróleo, plantas de almacenaje, gasoductos, plantas de compresión, separación, tratamiento de gas, redes de captación.

En el gráfico anterior podemos observar que de la evolución de la inversión en dichos sectores se pueden reconocer 2 momentos claves:

- En el verano del 2008 el precio del barril de petróleo alcanza un precio récord de casi 150 dólares. Justamente en dicho año se alcanza en Argentina un nivel de inversión de 3.202,33 millones de dólares, un 20\% más que en el año anterior. Pero luego surge una crisis financiera que se origina en un comienzo por una desconfianza crediticia en hipotecas (Subprime) en Estados Unidos (2007) y luego se expande a todo el mundo mediante la caída de la bolsa de valores (2008) ya que importantes entidades bancarias y grandes fondos de inversión tenían comprometidos sus activos en hipotecas, lo que provoca un freno en la inversión en el petróleo y el gas en el país, el cual se refleja en el año 2009.

- Se puede apreciar un repunte de las inversiones en el 2012 hasta la actualidad, lo cual coincide con la presentación del proyecto de ley de Soberanía hidrocarburífera de la República Argentina (2012) por el Poder Ejecutivo y su posterior aprobación en ambas cámaras, logrando así el traspaso de la propiedad de la empresa YPF al Estado Nacional.

Se observa una tendencia positiva en las inversiones en este tipo de energía en los últimos años hasta el 2016 en el que alcanza su punto máximo de inversión (10.775 millones de dólares) de los últimos 10 años, aprovechando el cambio de gobierno y las renovadas expectativas en el mismo.

Dicha situación cambiaría en el año 2017, el cual se observa en una fuerte disminución de la inversión en el país de casi un 38\% (10.775 a 6.696 millones de dólares).

\section{CONCLUSIONES}

En base al análisis del presente informe podemos decir, que la producción tanto de gas como de petróleo ha disminuido con el paso de los años.

En el caso de la producción de gas nacional detectamos una caída de medio punto en el año 2014, lo cual es un hecho histórico ya que nos demostró que la tendencia pudo cambiar, siendo esta positiva para los dos años siguiente. Para esto habrá que ver el atractivo que tenga el sector durante este año 2018 ya que para el año 2017 se podría decir que registró una pequeña baja de menos de medio punto.

A su vez observamos que el nivel de inversión va depender de lo que la YPF estatal decida invertir en el sector ya que es la empresa líder del sector.

Del mismo modo comprendemos que el sector a nivel mundial no se mantiene constante, observamos una variación y caída en el precio del petróleo, empresas que reducen sus inversiones o se retiran del mercado, cambio en las políticas de gobierno de los Estados Unidos.

En el caso de Argentina las estrategias que implemente el gobierno jugará un papel importante en la Economía, todo esto hace que el tablero mundial y en especial el de Argentina, en lo que respecta al petróleo y gas cambie continuamente. 


\section{RECOMENDACIONES}

Observamos en la provincia de Santa Cruz actividades de producción primaria y extractivas, como las que hemos estudiado, las cuales están organizadas en enclaves de alta productividad, pero con escaso valor agregado, es por ello que debemos:

- Aprovechar y fomentar el desarrollo industrial de la región con la energía que se dispondrá de la producción de la central termoeléctrica de Río Turbio, con la reserva fría de Río Chico, y la futura producción de las recientemente iniciadas represas hidroeléctricas Néstor Kirchner y Jorge Cepernic.

- Dicho Desarrollo Industrial podrá de alguna manera "descomprimir" el Estado Provincial, el cual es considerado el mayor empleador de la Provincia de Santa Cruz, generando empleo genuino y de valor agregado para la población.

- Desarrollo de políticas, tanto públicas como privadas para potenciar las ventajas comparativas existentes en la región.

\section{AGRADECIMIENTOS}

Al Director y al Co-Director del Proyecto de Investigación, Mg. Daniel Schinelli y Mg. Carlos Vacca respectivamente, por el acompañamiento durante todo este proceso de construcción de conocimiento científico y a los integrantes del proyecto 29/A310 por la colaboración con opiniones y por sus experiencias compartidas.

\section{ANEXOS}

$\underline{\text { Anexo 1: }}$

\begin{tabular}{|c|c|}
\hline \multicolumn{2}{|c|}{$\begin{array}{c}\text { Producción de Gas } \\
\text { Nacional }\end{array}$} \\
\hline Año & Miles de m3 \\
\hline 2000 & 45.211 .565 \\
\hline 2001 & 48.038 .567 \\
\hline 2002 & 46.457 .100 \\
\hline 2003 & 50.947 .741 \\
\hline 2004 & 52.156 .988 \\
\hline 2005 & 51.278 .599 \\
\hline 2006 & 51.645 .774 \\
\hline 2007 & 50.971 .152 \\
\hline 2008 & 50.488 .255 \\
\hline 2009 & 48.419 .249 \\
\hline 2010 & 47.107 .584 \\
\hline 2011 & 45.527 .554 \\
\hline 2012 & 44.123 .694 \\
\hline 2013 & 41.708 .289 \\
\hline 2014 & 41.484 .024 \\
\hline
\end{tabular}




\begin{tabular}{|l|l|}
2015 & 42.905 .533 \\
\hline 2016 & 44.987 .754 \\
\hline 2017 & 44.595 .411 \\
\hline
\end{tabular}

Anexo 2:

\begin{tabular}{|c|c|}
\hline \multicolumn{2}{|c|}{$\begin{array}{c}\text { Producción Petróleo } \\
\text { Nacional }\end{array}$} \\
\hline Año & m3 \\
\hline 2000 & 43.288 .766 \\
\hline 2001 & 44.616 .993 \\
\hline 2002 & 43.960 .582 \\
\hline 2003 & 42.957 .054 \\
\hline 2004 & 40.432 .948 \\
\hline 2005 & 38.572 .465 \\
\hline 2006 & 38.346 .334 \\
\hline 2007 & 37.904 .567 \\
\hline 2008 & 37.592 .946 \\
\hline 2009 & 36.239 .941 \\
\hline 2010 & 35.413 .416 \\
\hline 2011 & 33.326 .279 \\
\hline 2012 & 33.139 .519 \\
\hline 2013 & 32.461 .091 \\
\hline 2014 & 30.019 .610 \\
\hline 2015 & 29.692 .628 \\
\hline 2016 & 28.454 .403 \\
\hline 2017 & 26.554 .839 \\
\hline
\end{tabular}

Anexo 3:

\begin{tabular}{|c|c|}
\hline \multicolumn{2}{|c|}{$\begin{array}{c}\text { Inversiones en Petróleo y } \\
\text { Gas }\end{array}$} \\
\hline Año & Millones de USD \\
\hline 2005 & 145188,44 \\
\hline 2006 & 2220,89 \\
\hline 2007 & 2660,48 \\
\hline 2008 & 3202,33 \\
\hline 2009 & 2031,51 \\
\hline 2010 & 2640,71 \\
\hline 2011 & 3553,91 \\
\hline 2012 & 3415,66 \\
\hline 2013 & 5676,83 \\
\hline 2014 & 7734,53 \\
\hline 2015 & 8586,33 \\
\hline 2016 & 10775,03 \\
\hline 2017 & 6696,4 \\
\hline
\end{tabular}




\section{REFERENCIAS}

Diario INFOBAE: http://www.infobae.com/2015/02/26/1629439-otra-fuerte-caida-del-preciodel-petroleo

Diario El Patagónico On-Line: http://www.elpatagonico.net/nota/256392/

Diario El Litoral: http://www.ellitoral.com/index.php/diarios/2013/05/14/politica/POLI09.html

Diario El Mundo: http://www.elmundo.es/especiales/2008/10/economia/crisis2008/lascausas/

KOZULJ, R. (2015). "El Sector Energético Argentino: un análisis integrado de sus problemas, impactos y desafíos macroeconómicos.”; Viedma, Chubut; Editorial UNRN.

DI SBROIAVACCA, N. (2012). "La renta petrolera y su destino"; San Carlos de Bariloche, Río Negro; Editorial IAE.

Página web oficial de la Secretaría de Energía de la Nación (sistema SESCO): http://www.energia.gov.ar

Página web oficial del Instituto Argentino de la Energía: http://web.iae.org.ar

RECALDE, M. (2016). Una visión integral del sector energético Argentino: De las causas a las consecuencias de la ausencia de política energética de largo plazo. Págs. 15-38; Editorial Dunken.

Revista de Noticias e Investigación Café Babel: http://www.cafebabel.es/politica/articulo/la-crisis-economica-de-2008-explicada.html

SCHINELLI, D. y VACCA, C. (1999). "Reestructuración económica y su impacto en la provincia de Santa Cruz" en Salvia, A. (compilador) La Patagonia de los Noventa. Sectores que ganan, sociedades que pierden; Buenos Aires; Editorial Colmena.

SCHINELLI, D. y VACCA, C. (2007). "Hacia una transformación económica en la patagonia austral. La actividad turística en la nueva dinámica productiva y su impacto en el empleo" Revista Estudios Regionales No 3. Bs. As., CIPSA.

SCHINELLI, D. y VACCA, C. (2015). "La diversificación productiva en Santa Cruz para la superación del modelo de estado rentista."; Río Gallegos, Santa Cruz; Editorial UNPA. 